\title{
Model Predictive Control of a Class of Plants Subject to Random Disturbance
}

\author{
CHAO WANG and AKIRA OHSUMI \\ Department of Mechanical \& System Engineering, Graduate School of \\ Engineering and Science, Kyoto Institute of Technology, \\ Matsugasaki, Sakyo, Kyoto 606-8585, Japan \\ E-mail: ohsumi@ipc.kit.ac.jp
}

\begin{abstract}
In this paper, an optimal model predictive control (MPC) method is proposed for a class of high order plants which are modeled by first-order linear systems with timedelay. First, the mathematical model of the plant is described by the CARIMA model. Secondly, based on the state space model the optimal MPC is obtained incorporated with Kalman predictor/filter to get the present and future estimates of the state. Finally, a simulation example is given for comparing our method with the generalized predictive control method.
\end{abstract}

Keywords: Model Predicitve Control, Kalman estimator, Optimization with constaint

\section{Introduction}

In the past two decades the predictive control has achieved a significant level of maturity and acceptability with industrial success in practical process control applications (e.g. [1], [2]). Currently, it seems to be one of the most active topics in the field of process control engineering and it still extends the interesting fields.

Up to the present time, various methods such as MAC (Model Algorithmic Control), DMC (Dynamic Matrix Control), IDCOM (Identification and Command) and so forth have been proposed for the model predictive control (MPC). As one of the predictive controls, the generalized predictive control (GPC) algorithm have been widely used [3], [4]. The GPC algorithm is obtained, based on the CARIMA (Controller Auto-Regressive and Integrated Moving-Average) models, by minimizing a quadratic cost function which is sometimes called the receding horizon cost function and employed in the RHTC (Receding Horizon Tracking Control) [5].

To the authors' knowledge, the stochastic approach seems not to have been employed so ex- tensively in MPC, disregarding the fact that the actual plants have necessarily subjected to random disturbance and further the measurements are corrupted by random noise. For instance, although in the formulation of the GPC by Clarke, et al. [3], [4] the random disturbance is considered in the process model which is described by the CARIMA model, neither state predictor nor filtering is employed. It will be needless to say that any estimator should be incorporated with the algorithm of MPC to obtain an efficient predictive control. Lee, Morari, and Garcia [6] consider the MPC based on the state space model from the stochastic optimal control and incorporate it with the Kalman filter for the current state estimate, however the predictions of the future states are derived under the assumption that the future controls are absent, and any constraints which should be imposed on the control and/or state are ignored.

Most of MPCs are conceptually similar to the familiar LQ or LQG control in the formulation. However these control design methods have different points. One of the most important points 
which discriminate between these two formalisms is the existence of constraints on the control input and/or output [2].

In this paper, a MPC problem is considered for a typical class of plants which are subjected to random disturbance. Using the state space representation and the Kalman filter and predictor, the control problem is investigated for the quadratic cost function with receding horizon under input and output constraints.

\section{Model of the Plant}

Most of industrial processes are composed of many dynamic elements, usually first-order, so the full model may be described by a linear model of high-order. Such models would be very difficult to use and treat for control purposes, however, fortunately these models can be approximated by the simplified model consisting of a first-order process combined with a time delay element whose transfer function is given by [7]

$$
G(s)=\frac{K}{1+T s} e^{-L s},
$$

where $K$ is the process gain, $T$ is the time constant, and $L$ is the time-delay (Fig. 1).

To obtain the corresponding discrete-time transfer function, let $\tau$ be a sampling time and let

$$
a=e^{-\tau / T}, \quad b=K(1-a),
$$

provided that the time delay $L$ is an integer multiple of $\tau$, i.e., $L=d \tau$, or

$$
d=\frac{L}{\tau} .
$$

Then the discrete-time transfer function corresponding to (1) becomes [8]

$$
G(z)=\frac{b z^{-1}}{1-a z^{-1}} z^{-d} .
$$

Assuming the random disturbance $\{w(k)\}$ to the plant, this transfer function leads us to the CARIMA model,

$$
\left(1-a z^{-1}\right) y(k)=b z^{-d} u(k-1)+\frac{w(k)}{\Delta},
$$

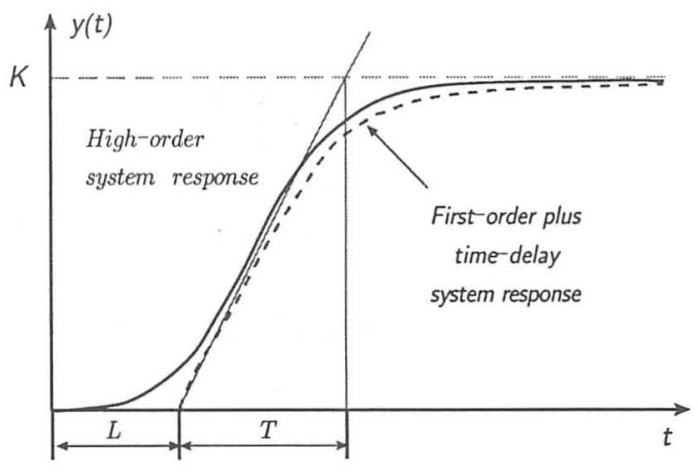

Fig.1. Step response of real process.

where $u(k)$ and $y(k)$ are the control and output sequences of the plant, and $\Delta=1-z^{-1}$. Since random disturbances can be regarded as random steps, in order to achieve offset-free closed-loop behavior given by these disturbances the controller must possess inherent integral action. For this reason the CARIMA model is often adopted in the MPC problems [3], [8].

Equation (4) can be transformed to:

$$
\begin{gathered}
y(k+1)=(1+a) y(k)-a y(k-1) \\
+b \Delta u(k-d)+w(k+1),
\end{gathered}
$$

where $\Delta u(k)=u(k)-u(k-1)$. In this paper the random disturbance $\{w(k)\}$ is modeled by a white Gaussian sequence with zero-mean and variance parameter $\sigma^{2}$.

The CARIMA model (5) can be represented in the observable canonical state space form:

$$
\begin{aligned}
x(k+1) & =A x(k)+B \Delta u(k)+G w(k) \\
y(k) & =C x(k)+w(k),
\end{aligned}
$$

where $x(k) \in R^{d+1}$ and

$$
\begin{gathered}
A=\left[\begin{array}{cccc}
1+a & & \\
-a & & I_{d} & \\
0 & & & \\
\vdots & & & \\
0 & 0 & \cdots & 0
\end{array}\right], B=\left[\begin{array}{c}
0 \\
\vdots \\
0 \\
b
\end{array}\right] \\
G=\left[\begin{array}{c}
1+a \\
-a \\
0 \\
\vdots \\
0
\end{array}\right], \quad C=\left[\begin{array}{llll}
1 & 0 & \cdots & 0
\end{array}\right] .
\end{gathered}
$$




\section{$3 \quad j$-Step Prediction}

Let $k$ be the present time step, and let $Y_{k}=$ $\{y(i), i=0,1, \cdots, k\}, U_{p k}=\{\Delta u(i), i=0,1, \cdots$, $k-1\}$ be the observation data up to $k$ and the past sequence of control increments, respectively. Then the $j$-step ahead prediction of the state $x(k+j)$, $\hat{x}(k+j \mid k)=\mathcal{E}\left\{x(k+j) \mid Y_{k}, U_{p k}\right\}$, is generated by the Kalman predictor:

$$
\hat{x}(k+j+1 \mid k)=A \hat{x}(k+j \mid k)+B \Delta u(k),
$$

and its predicted output is given by

$$
\hat{y}(k+j \mid k)=C \hat{x}(k+j \mid k) .
$$

From (8) and (9) the $N$-step ahead predictions of the output $y(\cdot)$ can be obtained as follows:

$$
\begin{aligned}
\hat{y}(k \mid k)= & C \hat{x}(k \mid k) \\
\hat{y}(k+1 \mid k)= & C \hat{x}(k+1 \mid k) \\
= & C A \hat{x}(k \mid k)+C B \Delta u(k) \\
\hat{y}(k+2 \mid k)= & C \hat{x}(k+2 \mid k) \\
= & C A^{2} \hat{x}(k \mid k)+C A B \Delta u(k) \\
& +C B \Delta u(k+1) \\
& \vdots \\
\hat{y}(k+N \mid k)= & C \hat{x}(k+N \mid k) \\
= & C A^{N} \hat{x}(k \mid k)+C A^{N-1} B \Delta u(k) \\
& +C A^{N-2} B \Delta u(k+1)+\cdots \\
& +C B \Delta u(k+N-1) .
\end{aligned}
$$

We write this as

where

$$
\hat{Y}_{k}=\Gamma_{N} \hat{x}(k \mid k)+H_{N} U_{k},
$$

$$
\begin{aligned}
& \hat{Y}_{k}=\left[\begin{array}{c}
\hat{y}(k+1 \mid k) \\
\hat{y}(k+2 \mid k) \\
\vdots \\
\hat{y}(k+N \mid k)
\end{array}\right], U_{k}=\left[\begin{array}{c}
\Delta u(k) \\
\Delta u(k+1) \\
\vdots \\
\Delta u(k+N-1)
\end{array}\right] \\
& \Gamma_{N}=\left[\begin{array}{c}
C A \\
C A^{2} \\
\vdots \\
C A^{N}
\end{array}\right] \\
& H_{N}=\left[\begin{array}{cccc}
C B & & & 0 \\
C A B & C B & & \\
\vdots & & \ddots & \\
C A^{N-1} B & \cdots & C A B & C B
\end{array}\right] \text {. }
\end{aligned}
$$

Equation (11) shows that every prediction of the future outputs is given by a linear function of the current estimate $\hat{x}(k \mid k)$ and (future) control increments.

Based on the state and observation models (6) and (7) where the random disturbances are the same, the estimation of the current state of $x(k)$ is obtained by the Kalman filter as: $(i=0,1, \cdots, k)$

$$
\begin{gathered}
\hat{x}(i+1 \mid i)=(A-G C) \hat{x}(i \mid i)+B \Delta u(i) \\
+G y(i) \\
\hat{x}(i \mid i)=\hat{x}(i \mid i-1)+K(i)\{y(i) \\
-C \hat{x}(i \mid i-1)\},
\end{gathered}
$$

where

$$
\begin{aligned}
& K(i)=P(i \mid i-1) C^{T}\left[C P(i \mid i-1) C^{T}+Q\right]^{-1} \\
& P(i+1 \mid i)=(A-G C) P(i \mid i)(A-G C)^{T} \\
& P(i \mid i)=P(i \mid i-1)-K(i) C P(i \mid i-1)
\end{aligned}
$$

with a priori given initial values $\hat{x}(0 \mid-1)=\hat{x}_{0}$ and $P(0 \mid-1)=P_{0}$, and $Q=\sigma^{2} I_{d+1}$.

\section{Reference Trajectory}

One of the advantages of predictive control is well known to react without any delay effect in the response before the change of the plant. In order to perform efficiently such behavior, the idea of reference trajectory is employed in the majority of methods for predictive controls. The reference trajectory is normally set as a smooth approximation from the current value of the output $y(k)$ towards the known reference.

To fix the idea, let $\{r(k)\}$ be the designed setpoint trajectory, and consider a reference trajectory which is tracked to achieve smooth trajectory of the plants,

$$
y_{r}(k+j)=r(k+j)+\alpha^{k}\{y(k)-r(k)\} .
$$

Here, in order to minimize the effect of random disturbance, instead of this, we consider the following reference trajectory in which the "raw" current observation $y(k)$ is replaced by its estimate, $\hat{y}(k \mid k)=C \hat{x}(k / k)$, 


$$
\begin{aligned}
y_{r}(k+j)= & \alpha^{k} C \hat{x}(k \mid k) \\
& +\left\{r(k+j)-\alpha^{k} r(k)\right\},
\end{aligned}
$$

where $\alpha$ is a parameter set between 0 and 1 .

\section{Optimal Control under Constraints}

Define an $N$-vector $Y_{r}$ for every $k$ by

$$
Y_{r}=\left[\begin{array}{c}
y_{r}(k+1) \\
y_{r}(k+2) \\
\vdots \\
y_{r}(k+N)
\end{array}\right]
$$

and consider a quadratic cost functional for the future $N$ steps:

$$
\begin{gathered}
J_{N}\left(U_{k}\right)=\left(\hat{Y}_{k}-Y_{r}\right)^{T} Q_{y}\left(\hat{Y}_{k}-Y_{r}\right) \\
+U_{k}^{T} R_{u} U_{k},
\end{gathered}
$$

where $Q_{y}$ and $R_{u}$ are symmetric, constant, positive definite weighting matrices. This is rewritten as

$$
\begin{aligned}
J_{N}\left(U_{k}\right)= & {\left[\Gamma_{N} \hat{x}(k \mid k)-Y_{r}\right]^{T} Q_{y}\left[\Gamma_{N} \hat{x}(k \mid k)-Y_{r}\right] } \\
& +2\left[\Gamma_{N} \hat{x}(k \mid k)-Y_{r}\right]^{T} Q_{y} H_{N} U_{k} \\
& +U_{k}^{T}\left(H_{N}^{T} Q_{Y} H_{N}+R_{u}\right) U_{k} .
\end{aligned}
$$

The control vector $U_{k}$ minimizing this cost functional can be determined uniquely. However, because of constructive safety and/or operational reasons, certain constraints should be imposed on the control and the output.

To do this, at the current step $k$ the following constraints are imposed for $j=0,1, \cdots, N-1$ :

$$
\left.\begin{array}{c}
u_{\min } \leq u(k+j) \leq u_{\max } \\
\Delta u_{\min } \leq \Delta u(k+j) \leq \Delta u_{\max } \\
y_{\min } \leq \hat{y}(k+j \mid k) \leq y_{\max }
\end{array}\right\}
$$

where $u_{*}, \Delta u_{*}$ and $y_{*}$ are preassigned upper or lower bounds. These constraints can be expressed in the vector forms as

$$
\left.\begin{array}{rl}
1 u_{\min } \leq T U_{k}+1 u(k-1) & \leq 1 u_{\max } \\
1 u_{\min } \leq U_{k} & \leq 1 \Delta u_{\max } \\
1 y_{\min } \leq H_{N} U_{k}+\Gamma_{N} \hat{x}(k \mid k) & \leq 1 y_{\max }
\end{array}\right\}
$$

where 1 and $T$ are $N$-vector and $N \times N$-lower triangular matrix whose entries are all ones. From

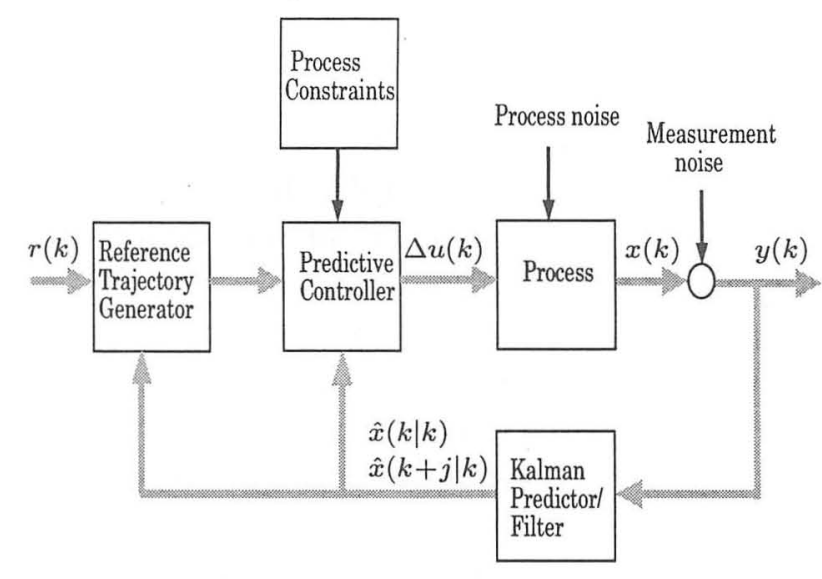

Fig.2. Step response of real process.

(22) the following linear vector inequality is obtained:

$$
R U_{k} \leq c_{k}
$$

where

$$
R=\left[\begin{array}{c}
I_{N \times N} \\
-I_{N \times N} \\
T \\
-T \\
H_{N} \\
-H_{N}
\end{array}\right], c_{k}=\left[\begin{array}{c}
1 \Delta u_{\max } \\
-1 \Delta u_{\min } \\
\mathbf{1} u_{\max }-1 u(k-1) \\
-1 u_{\min }+\mathbf{1} u(k-1) \\
\mathbf{1} y_{\max }-\Gamma_{N} \hat{x}(k \mid k) \\
-1 y_{\min }+\Gamma_{N} \hat{x}(k \mid k)
\end{array}\right] .
$$

Unfortunately, the problem of minimizing the quadratic cost functional (20) under a linear constraint such as (23) has no explicit solution; so we resort to solve it numerically as a convex quadratic programming for which efficient numerical methods exist. The optimal $\Delta u(k)$ is obtained as the first element of $U_{k}$.

The structure of the whole system is illustrated in Fig.2.

\section{A Simulation Study}

As a test plant to examine our purposed MPC method, a water purification plant is supposed which is considered as one of typical processes with large time-delay [9], [10]. In such a plant, raw water is promptly purified by injecting chemicals. The overall model of the water purification is so complex that it may be modeled by a firstorder system with time-delay as given by (1). The 
parameters of the transfer function were set as: $K=1, T=5$ and $L=10$. The sampling time $\tau$ was set as $\tau=1$. So, the plant parameters are: $a=0.8187, b=0.1813$, and $d=10$.

The variance parameter $\sigma^{2}$ of the random disturbance $w(k)$ was assumed to be $\sigma^{2}=1$. As for the reference trajectory, the setpoint reference was fixed to be $r(k)=40$ for all $k$ and $\alpha=0.68$; while for the cost criterion the matrixes $Q_{y}$ and $R_{u}$ were set $Q_{y}=\operatorname{diag}\{50,1, \cdots, 1\}$, and $R_{y}=$ $\operatorname{diag}\{1 / 3,1 / 3, \cdots, 1\}$.

The simulation results are shown in Figs. 3-6. The receding horizon was set $N=15$. In Figs. 3 and 4 the behavior of the output and input are depicted, comparing our MPC method with the GPC. The constraints imposed on the control and output are :

$$
\begin{aligned}
0 & \leq u(k+j) \leq 80 \\
-20 & \leq \Delta u(k+j) \leq 20 \\
0 & \leq \hat{y}(k+j \mid k) \leq 45
\end{aligned}
$$

for all $j=0,1,2, \cdots, N$. Comparing the sample paths, we can see that our MPC with constraints performs well. It should be noted that the GPC

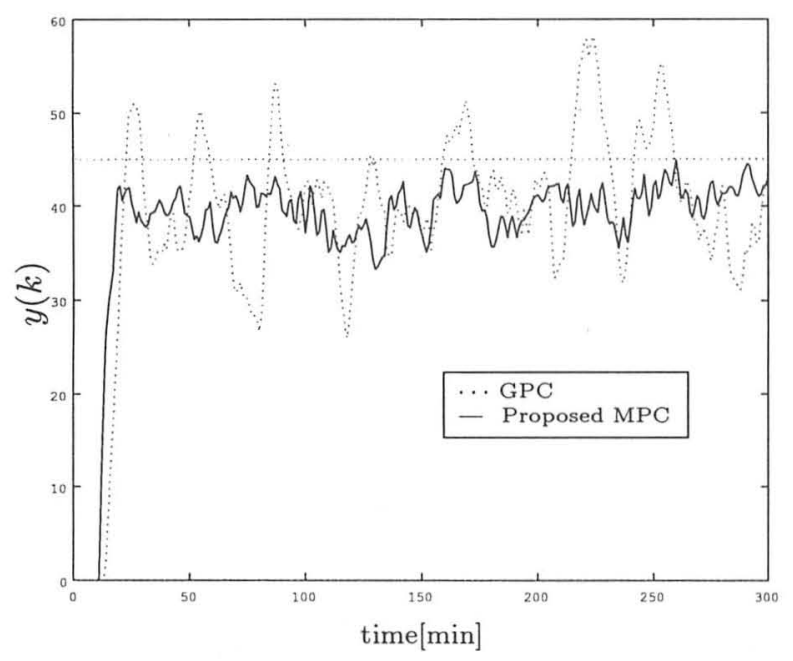

Fig.3. Comparison of sample paths of the output between the GPC and the proposed MPC methods. does not incorporated neither state estimator nor state and control constraints, while our MPC algorithm does both.

Figures 5 and 6 show how the constraints are satisfied by our MPC algorithm.

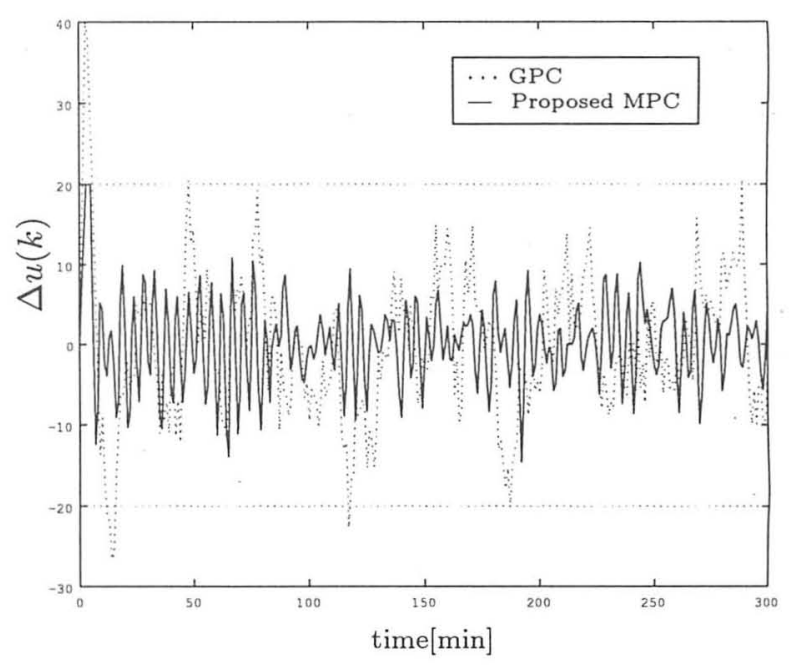

Fig.4. Comparison of sample paths of the input between the GPC and the proposed MPC methods.

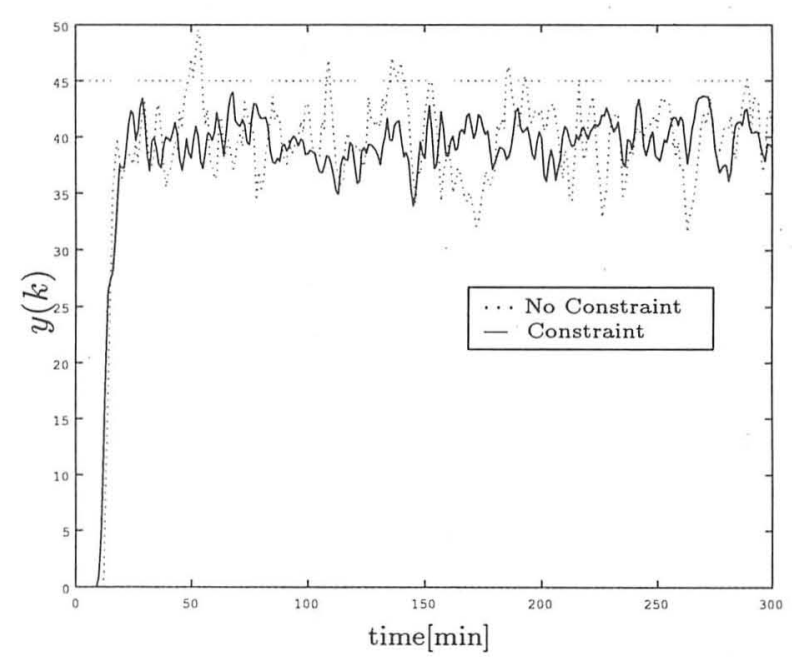

Fig.5. Comparison of sample paths of the controlled output $y(k)$. Two dotted straight lines depict the upper and lower constraint bounds. 


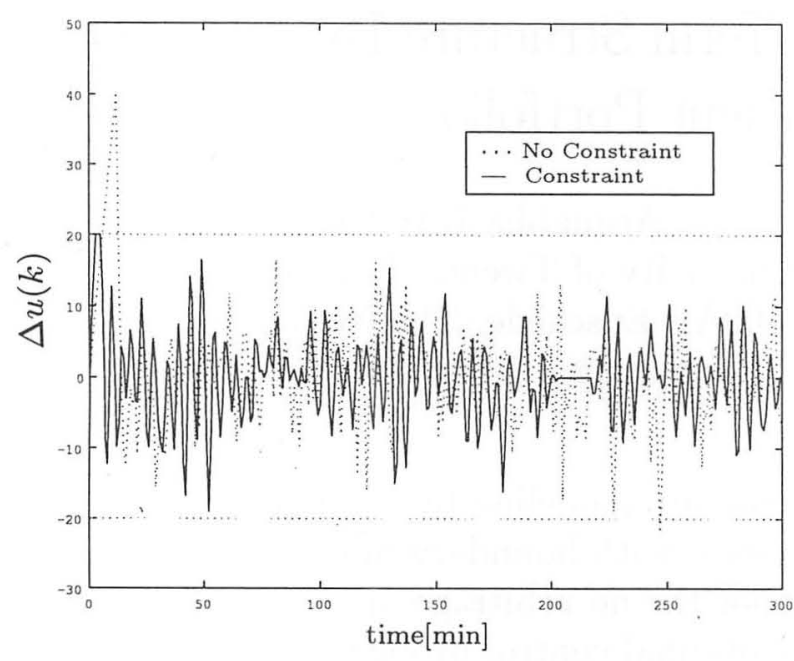

Fig.6. Comparison of sample paths of the control increment $\Delta u(k)$. Two straight lines depict the upper and lower bounds.

\section{Conclusion}

For a class of plants subjected to random disturbance, an efficient approach to the MPC has been proposed based on the CARIMA model by incorporating the Kalman filter and predictor to estimate the current and future plant state. By taking into account of the state and control constraints, the robustness of the control is necessarily guaranteed as contrasted with the conventional GPC, even for high intensity random disturbance.

Acknowledgment-The second author would like to thank Prof. M. Ohshima of Kyoto University who provided him the reference [2].

\section{References}

[1] J. Richalet: Industrial Applications of Model Based Predictive Control, Automatica, vol.29, no.5, 1993, pp.1251-1274.

[2] M. Ohshima: Predictive Control, Lecture Note, Kyoto Univ., 1997 (in Japanese).

[3] D. W. Clarke, C. Mohtadi, and P. S. Tuffs: Generalized Predictive Control-Part I. The basic Algorithm, Automatica, vol.23, no.2, 1987, pp.137-148.

[4] D. W. Clarke, C. Mohtadi, and P. S. Tuffs: Generalized Predictive Control-Part II. Extensions and Interpretations, Automatica, vol.23, no.2, 1987, pp.149-160.

[5] W. H. Kwon, and D. G. Byun: Receding Horizon Tracking Control as a Predictive Control and its Stability Results, Int. J. Control, vol.50, 1989, pp.1807-1824.

[6] J. H. Lee, M. Morari and C. E. Garcia: Statespace Interpretation $\mathrm{f}$ Model Predictive Control, Automatica, vol.30, no.4, 1994, pp.707717.

[7] D. E. Seborg, T. F. Edgnr, and D. A. Mellichamp: Process Dynamics and Control, John Wiley, N. Y., 1989.

[8] E. F. Camacho and C. Bordons: Model Predictive Control, Springer-Verlag, Berlin, 1999.

[9] M. Kubota, T. Ohto, and K. Kazama: Advanced Control of Coagulation in Water $\mathrm{Pu}$ rification Plant, Fuji-Jiho, vol.67, no.4, 1994, pp.231-236 (in Japanese)

[10] A. Ohsumi, T. Bamba, and T. Takatsu: Predictive Control of a Class of Plants Having Uncertainties, Proc. 44th ISCIE Annual Conference SCI2000, Kyoto, May, 2000, pp.265266. 